\title{
On finite-dimensional projections of distributions for solutions of randomly forced 2D Navier-Stokes equations
}

\author{
A. Agrachev ${ }^{\mathrm{a}, \mathrm{b}}$, S. Kuksin ${ }^{\mathrm{b}, \mathrm{c}}$, A. Sarychev ${ }^{\mathrm{d}}$, A. Shirikyan ${ }^{\mathrm{e}, *}$ \\ a SISSA-ISAS, via Beirut 2-4, Trieste 34014, Italy \\ b Steklov Institute of Mathematics, 8 Gubkina St., 117966 Moscow, Russia \\ ${ }^{\mathrm{c}}$ Department of Mathematics and Maxwell Institute for Mathematical Sciences, Heriot-Watt University, Edinburgh EH14 4AS, Scotland, UK \\ ${ }^{\mathrm{d}}$ DiMaD, University of Florence, via C. Lombroso 6/17, Firenze 50134, Italy \\ e Département de Mathématiques, Université de Cergy-Pontoise, Site de Saint-Martin, 2, avenue Adolphe Chauvin, \\ 95302 Cergy-Pontoise Cedex, France
}

Received 1 February 2006; accepted 19 June 2006

Available online 13 December 2006

\begin{abstract}
The paper is devoted to studying the image of probability measures on a Hilbert space under finite-dimensional analytic maps. We establish sufficient conditions under which the image of a measure has a density with respect to the Lebesgue measure and continuously depends on the map. The results obtained are applied to the 2D Navier-Stokes equations perturbed by various random forces of low dimension.

(c) 2006 Elsevier Masson SAS. All rights reserved.

\section{Résumé}

L'article est consacré à l'étude de l'image des mesures de probabilité sur un espace hilbertien par des applications analytiques de dimension finie. On établit des conditions suffisantes sous lesquelles l'image d'une mesure possède une densité par rapport à la mesure de Lebesgue et dépend continûment de l'application. Les résultats obtenus s'appliquent aux équations de Navier-Stokes 2D perturbées par diverses forces aléatoires de dimension peu élevée.
\end{abstract}

(c) 2006 Elsevier Masson SAS. All rights reserved.

MSC: 35Q30; 60H15; 93C20

Keywords: 2D Navier-Stokes system; Analytic transformations; Random perturbations

\section{Introduction}

Let us consider the 2D Navier-Stokes equations on the torus $\mathbb{T}^{2} \subset \mathbb{R}^{2}$ :

$$
\dot{u}+(u, \nabla) u-v \Delta u+\nabla p=f(t, x), \quad \operatorname{div} u=0, \quad x \in \mathbb{T}^{2} .
$$

\footnotetext{
* Corresponding author.

E-mail addresses: Agrachev@sissa.it (A. Agrachev), S.B.Kuksin@ma.hw.ac.uk (S. Kuksin), Andrey.Sarychev@dmd.unifi.it (A. Sarychev), Armen.Shirikyan@u-cergy.fr (A. Shirikyan).
} 
Here $u=\left(u_{1}, u_{2}\right)$ and $p$ are unknown velocity field and pressure, $v>0$ is the viscosity, and $f$ is an external force. Eqs. (0.1) are supplemented with the initial condition

$$
u(0)=u_{0},
$$

where $u_{0}$ is a given function belonging to the space $H$ of divergence-free vector fields in $L^{2}\left(\mathbb{T}^{2}, \mathbb{R}^{2}\right)$. It is well known $[20,5,21]$ that if the right-hand side $f$ satisfies some mild regularity assumptions, then problem $(0.1),(0.2)$ has a unique solution $u$ in an appropriate functional class. Our aim is to study some qualitative properties of solutions in the situation when $f$ is a random process with a sufficiently non-degenerate distribution. More precisely, let us assume that $f$ is a stochastic process on the positive half-line $\mathbb{R}_{+}$with range in $L^{2}\left(\mathbb{T}^{2}, \mathbb{R}^{2}\right)$ such that the distribution of its restriction to any interval $[0, T]$ is a non-degenerate decomposable measure (see Condition $(\mathrm{P})$ in Section 1.1). One of the main results of this paper says that for any finite-dimensional subspace $F \subset H$ and any $t>0$ the distribution of the projection of $u(t)$ to $F$ has a density with respect to the Lebesgue measure. Similar properties are true in the case when $f$ is a white noise in time or a sum of independent identically distributed random forces. Furthermore, if the random dynamical system associated with (0.1) generates a Markov process, then the above-mentioned property is valid for any stationary distribution. These results are important since for a number of problems in pure and applied mathematics only finitely many Fourier components of a solution $u$ matter; these components correspond to a finitedimensional subspace of $H$.

The fact that a solution of a nonlinear SDE stirred by a degenerate noise has a continuous density against the Lebesgue measure is very well known. The proofs are usually based on the Malliavin calculus (see [17] and the references therein). There have been a few works in which various versions of the Malliavin calculus were developed for some stochastic PDE's (for instance, see [18,4,6,15,8,16]). In particular, it was proved in [16] that if $f$ is white noise in time and sufficiently non-degenerate in the space variables, then the distribution of the projection of solution for $(0.1),(0.2)$ to any finite-dimensional subspace has a smooth density with respect to the Lebesgue measure.

The approach of this paper is completely different and is based on the controllability of (0.1) in finite-dimensional projections and an abstract result on the image of probability measures under analytic mappings. We emphasise that our method does not use the Gaussian structure of the noise, and the proof is simpler and shorter compared to the papers quoted above. At the same time, if the force $f$ is white in time, and the results of those works apply, then our information on the density of the distribution for the projection of $u(t)$ is weaker than, say, that obtained in [16].

The paper is organised as follows. In Section 1, we have compiled some preliminary results on decomposable measures on Hilbert spaces. Section 2 contains two abstract results on the transformation of probability measures under analytic mappings. In Section 3, we apply them to the 2D Navier-Stokes equations with different types of additive noise. Finally, in Appendix A, we prove some auxiliary results used in the main text.

\subsection{Notation}

Let $X$ be a Polish space, i. e., separable complete metric space. We denote by $B_{X}(a, R)$ the closed ball in $X$ of radius $R$ centred at $a$. If $a$ coincides with a selected point $\mathbf{0} \in X$, then we write $B_{X}(R)$. Let $\mathcal{B}(X)$ be the Borel $\sigma$-algebra on $X$ and let $\mathcal{P}(X)$ be the family of probability measures on $(X, \mathcal{B}(X))$. The space $\mathcal{P}(X)$ is endowed with the total variation norm:

$$
\left\|\mu_{1}-\mu_{2}\right\|_{\mathrm{var}}:=\sup _{\Gamma \in \mathcal{B}(X)}\left|\mu_{1}(\Gamma)-\mu_{2}(\Gamma)\right|, \quad \mu_{1}, \mu_{2} \in \mathcal{P}(X) .
$$

If $\mu_{1}, \mu_{2} \in \mathcal{P}(X)$ and $\mu_{1}$ is absolutely continuous with respect to $\mu_{2}$, then we write $\mu_{1} \ll \mu_{2}$. For a random variable $\xi$, we denote by $\mathcal{D}(\xi)$ its distribution.

For any Banach space $X$, we denote by $\|\cdot\|_{X}$ the norm in $X$. If $Y$ is another Banach space, then $\mathcal{L}(X, Y)$ stands for the space of bounded linear operators from $X$ to $Y$. In the case $X=Y$, we shall write $\mathcal{L}(X)$. If $X$ is finite-dimensional, then $\ell_{X}$ denotes the Lebesgue measure on $X$.

Let $J \subset \mathbb{R}$ be a closed interval and let $\mathbb{R}_{+}=[0,+\infty)$. We use the following functional spaces.

- $C_{b}(X)$ is the space of bounded continuous functions $f: X \rightarrow \mathbb{R}$ endowed with the norm

$$
\|f\|_{\infty}=\sup _{x \in X}|f(x)| .
$$


- $C(J, X)$ is the space of continuous functions $u: J \rightarrow X$.

- $L^{p}(J, X)$ is the space of Bochner-measurable functions $u: J \rightarrow X$ such that

$$
\|u\|_{L^{p}(J, X)}=\left(\int_{J}\|u(t)\|_{X}^{p} \mathrm{~d} t\right)^{1 / p}<\infty .
$$

- $L_{\text {loc }}^{p}\left(\mathbb{R}_{+}, X\right)$ is the space of functions $u: \mathbb{R}_{+} \rightarrow X$ whose restriction to any finite interval $J \subset \mathbb{R}_{+}$belongs to $L^{p}(J, X)$.

If $X$ is a Hilbert space and $F \subset X$ is a closed subspace, then $\mathrm{P}_{F}: X \rightarrow F$ denotes the orthogonal projection in $X$ onto $F$.

\section{Decomposable measures on Hilbert spaces}

\subsection{Definitions and examples}

Let $X$ be a separable Hilbert space with a scalar product $(\cdot, \cdot)$ and the corresponding norm $\|\cdot\|_{X}$. We denote by $\mathcal{B}(X)$ the Borel $\sigma$-algebra on $X$ and by $\mathcal{P}(X)$ the family of probability measures on $(X, \mathcal{B}(X))$.

Definition 1.1. We shall say that a measure $\mu \in \mathcal{P}(X)$ is decomposable if there is an orthonormal basis $\left\{g_{j}\right\} \subset X$ such that

$$
\mu=\bigotimes_{j=1}^{\infty} \mu_{j}
$$

where $\mu_{j}$ is the projection of $\mu$ to the one-dimensional space $X_{j}$ generated by $g_{j}$ and $\otimes$ denotes the tensor product of measures.

Example 1.2. Let $\mu \in \mathcal{P}(X)$ be a Gaussian measure (for instance, see [3]). It is well known that there is a vector $a \in X$ and a self-adjoint nuclear operator $K \in \mathcal{L}(X)$ such that the characteristic function of $\mu$ has the form

$$
\hat{\mu}(z)=\exp \left\{\mathrm{i}(a, z)-\frac{1}{2}(K z, z)\right\}, \quad z \in X .
$$

In this case, the vector $a$ is the mean value of $\mu$,

$$
a=\int_{X} x \mu(\mathrm{d} x),
$$

and $K$ is the covariance operator for $\mu$,

$$
(K z, z)=\int_{X}(z, x-a)^{2} \mu(\mathrm{d} x) ;
$$

we refer the reader to Chapter 2 in [3] for more details. Let $\left\{g_{j}\right\}$ be an orthonormal basis in $X$ formed of the eigenvectors of $K$ and let $\lambda_{j}$ be the eigenvalue of $K$ corresponding to $g_{j}$. If a vector $z \in X$ is written in the form

$$
z=\sum_{j=1}^{\infty} z_{j} g_{j}
$$

then relation (1.2) takes the form

$$
\hat{\mu}(z)=\prod_{j=1}^{\infty} \exp \left\{\mathrm{i}\left(a, g_{j}\right) z_{j}-\frac{1}{2} \lambda_{j} z_{j}^{2}\right\} .
$$


It follows that $\mu$ admits decomposition (1.1) in which $\mu_{j}$ is a one-dimensional Gaussian measure with mean value $\left(a, g_{j}\right)$ and variance $\lambda_{j}$. Note also that if $\lambda_{j}=0$ for some $j \geqslant 1$, then the measure $\mu$ is degenerate in the sense that its support is contained in a proper affine subspace of $X$.

In what follows, we shall deal with decomposable measures possessing some additional properties. Namely, we consider a measure $\mu \in \mathcal{P}(X)$ satisfying the following condition.

(P) The measure $\mu$ is decomposable and has a finite second moment

$$
\int_{X}\|x\|_{X}^{2} \mu(\mathrm{d} x)<\infty .
$$

Moreover, every measure $\mu_{j}$ in (1.1) possesses a continuous density $\rho_{j}$ with respect to the Lebesgue measure on $X_{j}$.

The following lemma describes the random variables whose distribution satisfies property $(\mathrm{P})$; its proof is obvious.

Lemma 1.3. The distribution of an $X$-valued random variable $\xi$ satisfies condition $(\mathrm{P})$ if and only if $\xi$ has the form

$$
\xi=\sum_{j=1}^{\infty} b_{j} \xi_{j} g_{j},
$$

where $\left\{g_{j}\right\}$ is an orthonormal basis in $X,\left\{\xi_{j}\right\}$ is a sequence of scalar independent random variables such that $\mathbb{E} \xi_{j}^{2}=1$ and the law of $\xi_{j}$ has a continuous density with respect to the Lebesgue measure, and $b_{j}>0$ are some constants satisfying the condition

$$
\sum_{j=1}^{\infty} b_{j}^{2}<\infty
$$

Example 1.4. Let $\mu \in \mathcal{P}(X)$ be a Gaussian measure with a mean value $a \in X$ and a covariance operator $K$. We claim that $\mu$ satisfies condition (P) if and only if it is non-degenerate, i.e., all eigenvalues of $K$ are positive. Indeed, Fernique's theorem (see [3]) implies that condition (1.4) is satisfied for any Gaussian measure. Furthermore, it follows from (1.3) that the projection $\mu_{j}$ of $\mu$ to $X_{j}$ is a one-dimensional Gaussian measure with variance $\lambda_{j}$. Thus, $\mu_{j}$ possesses a continuous density with respect to the Lebesgue measure if and only if $\lambda_{j}>0$.

For the sequel, we note that if $\mu \in \mathcal{P}(X)$ is a non-degenerate Gaussian measure, then

$$
\mu(B)>0 \text { for any ball } B \subset X \text {; }
$$

see Section 3.5 in [3] for a proof.

\subsection{Support of decomposable measures}

Let $X$ be a separable Hilbert space and let $\mu \in \mathcal{P}(X)$ be a measure possessing property (P). Denote by $\left\{g_{j}\right\}$ an orthonormal basis in $X$ for which representation (1.1) holds and, for any integer $N \geqslant 1$, define $X_{(N)}$ as the $N$-dimensional space spanned by $g_{j}, 1 \leqslant j \leqslant N$. Let

$$
\mu_{(N)}=\bigotimes_{j=1}^{N} \mu_{j}, \quad v_{(N)}=\bigotimes_{j=N+1}^{\infty} \mu_{j}
$$

If $a \in X_{(N)}$ and $R>0$, then we denote by $B_{N}(a, R)$ the closed ball in $X_{(N)}$ of radius $R$ centred at $a$. In the case $a=0$, we write $B_{N}(R)$.

Proposition 1.5. Let $\mu \in \mathcal{P}(X)$ be a measure satisfying condition $(\mathrm{P})$. Then there is a point

$$
A=\sum_{j=1}^{\infty} a_{j} g_{j} \in X
$$


such that ${ }^{1}$

$$
\rho_{j}\left(a_{j}\right)>0 \text { for all } j \geqslant 1 \text {. }
$$

Furthermore, if $A \in X$ is a point satisfying (1.10), then for any integer $N \geqslant 1$ there is $r_{N}>0$ such that

$$
\operatorname{supp} \mu \supset B_{N}\left(A_{N}, r_{N}\right), \quad A_{N}=\sum_{j=1}^{N} a_{j} g_{j}
$$

Proof. To prove the existence of $A$, denote by $A^{0}=\sum_{j} a_{j}^{0} g_{j}$ any point in the support of $\mu$. Then $a_{j}^{0} \in \operatorname{supp} \mu_{j}$ for any $j \geqslant 1$. Since $\mu_{j} \ll \ell_{X_{j}}$ (where $\ell_{X_{j}}$ denotes the Lebesgue measure on $X_{j}$ ), there is $a_{j} \in \mathbb{R}$ such that $\left|a_{j}-a_{j}^{0}\right| \leqslant j^{-1}$ and (1.10) holds. Defining $A \in X$ by relation (1.9), we obtain the required result.

When proving the second part of the proposition, we shall assume, without loss of generality, that $A=0$. Let us fix any integer $N \geqslant 1$. It follows from (1.8) and (1.10) that

$$
\operatorname{supp} \mu_{(N)} \supset B_{N}\left(r_{N}\right),
$$

where $r_{N}>0$ is sufficiently small. We claim that (1.11) holds for the same constant $r_{N}>0$. Indeed, let $x \in B_{N}\left(r_{N}\right)$ and $\varepsilon>0$. Consider an $X$-valued random variable $\xi$ with distribution $\mu$. By Lemma 1.3, it has the form (1.5). We wish to show that

$$
\mathbb{P}\left\{\|\xi-x\|_{X} \leqslant \varepsilon\right\}>0 .
$$

To this end, define

$$
\varphi_{N}=\sum_{j=1}^{N} b_{j} \xi_{j} g_{j}, \quad \psi_{N}=\sum_{j=N+1}^{\infty} b_{j} \xi_{j} g_{j} .
$$

It is clear that (1.13) will be established if we prove that

$$
\mathbb{P}\left\{\left\|\varphi_{N}-x\right\|_{X} \leqslant \varepsilon / 2\right\}>0, \quad \mathbb{P}\left\{\left\|\psi_{N}\right\|_{X} \leqslant \varepsilon / 2\right\}>0 .
$$

The first inequality follows immediately from (1.12). To prove the second, choose an integer $M>N$ and write

$$
\left\|\psi_{N}\right\|_{X}^{2}=\sum_{j=N+1}^{M} b_{j}^{2} \xi_{j}^{2}+\sum_{j=M+1}^{\infty} b_{j}^{2} \xi_{j}^{2}=S_{M}+R_{M} .
$$

In view of (1.6) and the relation $\mathbb{E} \xi_{j}^{2}=1$, we have

$$
\mathbb{E} R_{M}=\sum_{j=M+1}^{\infty} b_{j}^{2} \rightarrow 0 \quad \text { as } M \rightarrow \infty .
$$

By Chebyshev's inequality, it follows that

$$
\mathbb{P}\left\{R_{M} \leqslant \delta\right\} \rightarrow 1 \quad \text { as } M \rightarrow \infty,
$$

where $\delta>0$ is an arbitrary constant. Furthermore, inequalities (1.10) with $a_{j}=0$ imply that, for any fixed $M>N$ and $\delta>0$, we have

$$
\mathbb{P}\left\{S_{M} \leqslant \delta\right\}>0 .
$$

Combining (1.15)-(1.17) and recalling that $R_{M}$ and $S_{M}$ are independent, we arrive at the second inequality in (1.14). This completes the proof of the proposition.

\footnotetext{
1 In what follows, we identify $X_{j}$ with the real line and regard $\rho_{j}$ as a function of a real variable.
} 


\subsection{A zero-one law for analytic functions}

Let $X$ be a separable Hilbert space and let $f: X \rightarrow \mathbb{R}$ be a continuous function. Recall that $f$ is said to be analytic if for any $x_{0} \in X$ there is $\delta>0$ such that

$$
f(x)=f\left(x_{0}\right)+\sum_{m=1}^{\infty} L_{m}\left(x-x_{0}\right) \quad \text { for } x \in B_{X}\left(x_{0}, \delta\right),
$$

where $L_{m}: X \rightarrow \mathbb{R}$ is an $m$-linear functional and the series in (1.18) converges regularly. The latter means that

$$
\sum_{m=1}^{\infty}\left\|L_{m}\right\| \delta^{m}<\infty
$$

where $\|\cdot\|$ stands for the norm of multilinear functionals:

$$
\left\|L_{m}\right\|=\sup _{\|x\|_{X} \leqslant 1}\left|L_{m} x\right| .
$$

We refer the reader to [13] or [21] for more details.

For any continuous function $f: X \rightarrow \mathbb{R}$, we set

$$
\mathcal{N}_{f}=\{x \in X: f(x)=0\} .
$$

It is well known that if $\operatorname{dim} X<\infty$ and $f$ is analytic, then either $\ell_{X}\left(\mathcal{N}_{f}\right)=0$ or $f \equiv 0$. The following theorem shows that a similar result is true in the infinite-dimensional case.

Theorem 1.6. Let $f: X \rightarrow \mathbb{R}$ be an analytic function and let $\mu \in \mathcal{P}(X)$ be a measure possessing property (P). Then

$$
\mu\left(\mathcal{N}_{f}\right)=0 \text { or } 1 .
$$

Furthermore, if $f$ is not identically zero, then $\mu\left(\mathcal{N}_{f}\right)=0$.

Proof. We shall need a result from [7]. To formulate it, let us introduce some definitions.

Recall that the one-dimensional spaces $X_{j}$ are defined in condition $(\mathrm{P})$ and that $X_{(N)}$ stands for the vector space spanned by $X_{j}, 1 \leqslant j \leqslant N$. Denote by $X_{(N)}^{\perp}$ the orthogonal complement of $X_{(N)}$ in $X$ and set $X_{(\infty)}=\bigcup_{N} X_{(N)}$. We shall say that $A \in \mathcal{B}(X)$ is a finite zero-one $\mu$-set if

$$
v_{(N)}\left(\left\{y \in X_{(N)}^{\perp}: \mu_{(N)}\left(A_{N}(y)\right)=0 \text { or } 1\right\}\right)=1 \text { for any integer } N \geqslant 1,
$$

where $A_{N}(y)=\left\{x \in X_{(N)}: x+y \in A\right\}$, and the measures $\mu_{(N)}$ and $\nu_{(N)}$ are defined by (1.8).

According to Theorem 4 in [7], if $\mu$ is a decomposable measure on $X$, then for any finite zero-one $\mu$-set $A \in \mathcal{B}(X)$ there is $A^{\prime} \in \mathcal{B}(X)$ such that

$$
A^{\prime}+X_{(\infty)}=A^{\prime}, \quad \mu(A)=\mu\left(A^{\prime}\right) .
$$

Applying the Kolmogorov zero-one law (see [9]), we see that $\mu\left(A^{\prime}\right)=0$ or 1 . Thus, the measure of any finite zero-one $\mu$-set is either zero or one.

To prove (1.19), note that if $f$ is analytic, then for any integer $N \geqslant 1$ and any $y \in X_{(N)}^{\perp}$, we have either $\ell_{N}\left(\mathcal{N}_{f}(y)\right)=0$ or $\ell_{N}\left(X_{(N)} \backslash \mathcal{N}_{f}(y)\right)=0$, where $\ell_{N}$ denotes the Lebesgue measure on $X_{(N)}$. Since $\mu_{(N)} \ll \ell_{N}$ (see condition $(\mathrm{P}))$, we see that

$$
\mu_{(N)}\left(\mathcal{N}_{f}(y)\right)=0 \text { or } 1 \quad \text { for any } y \in X_{(N)}^{\perp} .
$$

Thus, $\mathcal{N}_{f}$ is a finite zero-one $\mu$-set, and (1.19) follows from what has been said above.

We now suppose that $f \not \equiv 0$. In this case, there is $x_{0} \in X$ such that $f\left(x_{0}\right) \neq 0$, and since $\left\{g_{j}\right\}$ is a basis in $X$ and $f$ is continuous, there is no loss of generality in assuming that $x_{0} \in X_{(N)}$ for some integer $N \geqslant 1$. In view of (1.19), the required assertion will be established if we show that

$$
\mu(\{x \in X: f(x) \neq 0\}) \neq 0 .
$$


The restriction of $f$ to $X_{(N)}$ is an analytic function on a finite-dimensional space. It follows that

$$
f(x) \neq 0 \quad \text { for } \ell_{N} \text {-almost every } x \in X_{(N)} .
$$

Recalling Proposition 1.5, we can find a point $x_{0} \in \operatorname{supp} \mu$ such that $f\left(x_{0}\right) \neq 0$. By continuity, there is $\delta>0$ such that

$$
f(x) \neq 0 \text { for } x \in B\left(x_{0}, \delta\right) .
$$

This implies that $\{f(x) \neq 0\} \supset B\left(x_{0}, \delta\right)$, and therefore (1.20) holds.

\section{Image of measures under analytic maps}

\subsection{Formulations of the results}

Let $(U, d)$ be a metric space and let $X$ and $F$ be finite-dimensional vector spaces. Consider a continuous operator $f: U \times X \rightarrow F$. For any probability measure $\mu \in \mathcal{P}(X)$ and any $u \in U$, denote by $f_{*}(u, \mu)$ the image of $\mu$ under $f(u, \cdot)$.

Theorem 2.1. Suppose that, for any $u \in U$, the function $f(u, \cdot)$ is analytic and the interior of the set $f(u, X)$ is nonempty. Let $\mu \in \mathcal{P}(X)$ be a measure possessing a continuous density $\rho(x)$ with respect to the Lebesgue measure on $X$. Then the following assertions hold.

(i) For any $u_{0} \in U$, the measure $f_{*}\left(u_{0}, \mu\right)$ is absolutely continuous with respect to $\ell_{F}$.

(ii) The function $f_{*}(\cdot, \mu)$ from $U$ to the space $\mathcal{P}(F)$ endowed with the total variation norm is continuous.

Our next goal is to study the case in which $X$ is an infinite-dimensional space. More precisely, suppose that $X$ is a separable Hilbert space and $F$ is a finite-dimensional vector space. Recall that condition $(\mathrm{P})$ is introduced in Section 1.1.

Theorem 2.2. Let $f: U \times X \rightarrow F$ be a continuous function such that $f(u, \cdot)$ is analytic for any $u \in U$ and the derivative $D_{x} f(u, x)$ is continuous with respect to $(u, x)$. Suppose that, for any $u \in U$, there is a ball $B_{u}$ in a finitedimensional subspace $X_{u} \subset X$ such that the interior of the set $f\left(u, B_{u}\right)$ is non-empty. Then for any measure $\mu \in \mathcal{P}(X)$ satisfying condition (P) statements (i) and (ii) of Theorem 2.1 take place.

\subsection{Proof of Theorem 2.1}

Let us fix any point $u_{0} \in U$ and denote by $D_{x} f\left(u_{0}, x\right)$ the derivative (Jacobian) of the map $f\left(u_{0}, \cdot\right): X \rightarrow F$ at the point $x \in X$. We first show that the matrix $D_{x} f\left(u_{0}, x\right)$ has a minor $m(x)$ of the size $\operatorname{dim} F$ such that

$$
m(x) \neq 0 \quad \text { for } \mu \text {-almost every } x \in X \text {. }
$$

To this end, recall that a point $x \in X$ is said to be regular for $f\left(u_{0}, \cdot\right)$ if the rank of $D_{x} f\left(u_{0}, x\right)$ is maximal. Any point that is not regular is said to be singular. In view of Sard's theorem (see [19]), the image under the smooth function $f\left(u_{0}, \cdot\right)$ of the set of its singular points has zero Lebesgue measure. Since the interior of $f\left(u_{0}, X\right)$ is nonempty, we conclude that $f\left(u_{0}, \cdot\right)$ has a regular point $x_{0} \in X$.

Let $m(x)$ be the minor of $D_{x} f\left(u_{0}, x\right)$ that is non-zero at $x_{0}$. Since $m(x)$ is analytic, we see that $m(x) \neq 0$ almost everywhere with respect to the Lebesgue measure $\ell_{X}$. Since $\mu$ is absolutely continuous with respect to $\ell_{X}$, we conclude that (2.1) holds.

For any $\varepsilon>0$, we denote

$$
X^{\varepsilon}=\left\{x \in X:|x| \leqslant \varepsilon^{-1},|m(x)| \geqslant \varepsilon\right\} .
$$

Then

$$
v_{\varepsilon}:=\mu\left(X \backslash X^{\varepsilon}\right) \rightarrow 0 \quad \text { as } \varepsilon \rightarrow 0 .
$$

Let us take any $x \in X^{\varepsilon}$ and write it as $x=\left(x_{1}, x_{2}\right)$, where $x_{1}$ denotes the variables entering the minor $m(x)$. Accordingly, the space $X$ can be represented as a direct product $X=X_{1} \times X_{2}$. Applying the implicit function theorem, we 
can find open balls $V_{1} \subset X_{1}$ and $V_{2} \subset X_{2}$ such that for any $x_{2} \in V_{2}$ and $u \in B^{\varepsilon}=B_{X}\left(u_{0}, r_{\varepsilon}\right)$, the map $f\left(u, \cdot, x_{2}\right)$ is a diffeomorphism of the domain $V_{1}$ onto its image $W\left(u, x_{2}\right)$. Here $r_{\varepsilon}>0$ is a constant that goes to zero with $\varepsilon$. Accordingly, we can write $x_{1}$ in terms of $u \in B^{\varepsilon}, x_{2} \in V_{2}$ and $y=f\left(u, x_{1}, x_{2}\right) \in W\left(u, x_{2}\right)$ :

$$
x_{1}=g\left(u, y, x_{2}\right) .
$$

The sets $V=V_{1} \times V_{2}$ corresponding to various $x \in X^{\varepsilon}$ form an open cover of the compact set $X^{\varepsilon}$. Let us find a finite sub-cover $\left\{V^{j}\right\}$. We denote by $\left\{\varphi_{j}(x)\right\}$ a continuous partition of unity on $X^{\varepsilon}$ subordinate to $\left\{V^{j}\right\}$. That is, $\varphi_{j} \geqslant 0$, $\operatorname{supp} \varphi_{j} \subset V^{j}$, and $\left(\sum \varphi_{j}\right)(x)=1$ for $x \in X^{\varepsilon}$. Let $\mu_{j}=\varphi_{j} \mu$. Then

$$
\mu_{j}=\varphi_{j}(x) \rho(x) \mathrm{d} x_{1} \mathrm{~d} x_{2}=\varphi_{j}\left(x_{1}, x_{2}\right) \rho\left(x_{1}, x_{2}\right)\left|m\left(u, x_{1}, x_{2}\right)\right|^{-1} \mathrm{~d} y \mathrm{~d} x_{2},
$$

where $m(u, x)$ is the minor of $D_{x} f(u, x)$ corresponding to $x_{1}$, and $x_{1}=g\left(u, y, x_{2}\right)$ on the right-hand side of (2.2) Hence, $f_{*}\left(u, \mu_{j}\right)=g_{j}(u, y) \mathrm{d} y$, where

$$
g_{j}(u, y)=\int \tilde{\varphi}_{j}\left(y, x_{2}\right) \tilde{\rho}\left(y, x_{2}\right)\left|\tilde{m}\left(u, y, x_{2}\right)\right|^{-1} \mathrm{~d} x_{2}
$$

with $\tilde{\varphi}_{j}\left(y, x_{2}\right)=\varphi_{j}\left(g\left(u, y, x_{2}\right), x_{2}\right)$, etc. Let us denote $\mu_{\varepsilon}=\left(\sum \varphi_{j}\right) \mu$. Then $\left\|\mu-\mu_{\varepsilon}\right\|_{\text {var }} \leqslant v_{\varepsilon}$. We have

$$
f_{*}\left(u, \mu_{\varepsilon}\right)=g_{\varepsilon}(u, y) \mathrm{d} y,
$$

where $g_{\varepsilon}(u, y)=\sum g_{j}(u, y)$ is a continuous function of $u \in B^{\varepsilon}$ and $y$. Clearly,

$$
\left\|f_{*}\left(u, \mu_{\varepsilon}\right)-f_{*}(u, \mu)\right\|_{\mathrm{var}} \leqslant v_{\varepsilon} \quad \text { for any } u \in B^{\varepsilon} .
$$

Relations (2.3) and (2.4) with $u=u_{0}$ and $\varepsilon \rightarrow 0$ imply assertion (i). Indeed, for any measurable set $Q \subset F$ with zero Lebesgue measure, we have

$$
f_{*}(u, \mu)(Q)=\left(f_{*}(u, \mu)-f_{*}\left(u, \mu_{\varepsilon}\right)\right)(Q)+f_{*}\left(u, \mu^{\varepsilon}\right)(Q),
$$

so $f_{*}(u, \mu)(Q) \leqslant v_{\varepsilon}$ for each $\varepsilon$. Hence, $f_{*}(u, \mu)(Q)=0$.

To prove (ii), we fix any $\gamma>0$ and choose $\varepsilon>0$ such that $v_{\varepsilon}<\frac{1}{3} \gamma$. Due to (2.4), for $u \in B^{\varepsilon}$ we have

$$
\begin{aligned}
\left\|f_{*}(u, \mu)-f_{*}\left(u_{0}, \mu\right)\right\|_{\mathrm{var}} & \leqslant 2 v_{\varepsilon}+\left\|f_{*}\left(u, \mu_{\varepsilon}\right)-f_{*}\left(u_{0}, \mu_{\varepsilon}\right)\right\|_{\mathrm{var}} \\
& \leqslant 2 v_{\varepsilon}+\frac{1}{2} \int\left|g_{\varepsilon}(u, y)-g_{\varepsilon}\left(u_{0}, y\right)\right| \mathrm{d} y \leqslant \gamma,
\end{aligned}
$$

if $u$ is sufficiently close to $u_{0}$. Since $u_{0}$ is an arbitrary point, what has been said implies (ii).

Remark 2.3. Analysing the proof given above, one easily sees that, instead of assuming the existence of interior points for the set $f(u, X)$, we could require that the function $f(u, \cdot)$ should have at least one regular point for any $u \in U$ (cf. the beginning of the proof).

\subsection{Proof of Theorem 2.2}

Step 1. Let us fix any $u_{0} \in U$ and show that there is finite-dimensional subspace $X_{1} \subset X$ spanned by some vectors of the basis $\left\{g_{j}\right\}$ such that $\operatorname{dim} X_{1}=\operatorname{dim} F$ and (2.1) holds, where $m(x)$ denotes the determinant of the matrix for the restriction of $D_{x} f\left(u_{0}, x\right)$ to $X_{1}$. Indeed, by the hypothesis, there is a ball $B_{u_{0}}$ in a finite-dimensional subspace $X_{u_{0}} \subset X$ such that the interior of $f\left(u_{0}, B_{u_{0}}\right)$ is non-empty. Since $\left\{g_{j}\right\}$ is a basis in $X$, for any $\delta>0$ there is a finitedimensional subspace $Y \subset X$ spanned by some vectors of $\left\{g_{j}\right\}$ such that

$$
\left\|\mathrm{P}_{Y} y-y\right\|_{X} \leqslant \delta \quad \text { for any } y \in B_{u_{0}},
$$

where $\mathrm{P}_{Y}: X \rightarrow X$ is the orthogonal projection in $X$ onto the subspace $Y$. Now note that $f\left(u_{0}, \cdot\right)$ is continuous and $B_{u_{0}}$ is compact. Therefore for any $\varepsilon>0$ we can find $\delta>0$ such that

$$
\left\|f\left(u_{0}, z\right)-f\left(u_{0}, y\right)\right\|_{F} \leqslant \varepsilon \quad \text { for } y \in B_{u_{0}},\|z-y\|_{X} \leqslant \delta .
$$


Combining (2.5) and (2.6), we see that for any $\varepsilon>0$ there is a finite-dimensional subspace $Y \subset X$ spanned by some vectors of $\left\{g_{j}\right\}$ such that

$$
\left\|f\left(u_{0}, \mathrm{P}_{Y} y\right)-f\left(u_{0}, y\right)\right\|_{F} \leqslant \varepsilon \quad \text { for } y \in B_{u_{0}} .
$$

Choosing $\varepsilon>0$ sufficiently small and applying Proposition A.1 of the Appendix (see Appendix A), we conclude that the interior of the set $f\left(u_{0}, Y\right)$ is non-empty. Since $\operatorname{dim} Y<\infty$, Sard's theorem implies that the function $f\left(u_{0}, \cdot\right): Y \rightarrow F$ has at least one regular point $x_{0} \in Y$. Let us denote by $X_{1}$ a subspace spanned by some vectors of the basis $\left\{g_{j}\right\}$ such that the restriction of $D_{x} f\left(u_{0}, x_{0}\right)$ to $X_{1}$ is an isomorphism from $X_{1}$ onto $F$. Then $m\left(x_{0}\right) \neq 0$. Since $m(x)$ is an analytic function, the required result follows from Theorem 1.6.

Step 2. We now repeat the argument used in the proof of Theorem 2.1. Let us represent $X$ as the direct product $X=X_{1} \times X_{2}$, where $X_{1}$ is constructed in Step 1 and $X_{2}$ denotes the orthogonal complement of $X_{1}$ in $X$. Denote by $\lambda_{1}$ and $\lambda_{2}$ the projections of $\mu$ to the subspaces $X_{1}$ and $X_{2}$, respectively, and by $\rho\left(x_{1}\right)$ the density of $\lambda_{1}$ with respect to $\ell_{X_{1}}$. For any $\varepsilon>0$, let us choose a compact set $X^{\varepsilon} \subset X$ such that

$$
\|x\|_{X} \leqslant \varepsilon^{-1}, \quad|m(x)| \geqslant \varepsilon \quad \text { for } x \in X^{\varepsilon}, \quad v_{\varepsilon}:=\mu\left(X \backslash X^{\varepsilon}\right) \leqslant \varepsilon .
$$

As in the proof of Theorem 2.1, we can find a constant $r_{\varepsilon}>0$ going to zero with $\varepsilon$ and a finite cover $\left\{V^{j}=V_{1}^{j} \times V_{2}^{j}\right\}$ of the compact set $X^{\varepsilon}$ such that $V_{1}^{j} \subset X_{1}$ and $V_{2}^{j} \subset X_{2}$ are balls, and for any $x_{2} \in V_{2}$ and $u \in B^{\varepsilon}=B_{X}\left(u_{0}, r_{\varepsilon}\right)$, the map $f\left(u, \cdot, x_{2}\right)$ is a diffeomorphism of the domain $V_{1}$ onto its image $W\left(u, x_{2}\right)$. We denote by $x_{1}=g\left(u, \cdot, x_{2}\right)$ the inverse function of $f\left(u, \cdot, x_{2}\right)$. Let $\left\{\varphi_{j}(x)\right\}$ be a continuous partition of unity on $X^{\varepsilon}$ subordinate to $\left\{V^{j}\right\}$ and let $\mu_{j}=\varphi_{j} \mu$. Then we have (cf. (2.2))

$$
\mu_{j}=\varphi_{j}(x) \rho\left(x_{1}\right) \mathrm{d} x_{1} \lambda\left(\mathrm{d} x_{2}\right)=\varphi_{j}\left(x_{1}, x_{2}\right) \rho\left(x_{1}\right)\left|m\left(u, x_{1}, x_{2}\right)\right|^{-1} \mathrm{~d} y \lambda\left(\mathrm{d} x_{2}\right),
$$

where $m(u, x)$ denotes the determinant of the restriction of $D_{x} f(u, x)$ to $X_{1}$, and $x_{1}=g\left(u, y, x_{2}\right)$ on the right-hand side of the formula. The rest of the proof is literally the same as that of Theorem 2.1, and therefore we omit it.

Remark 2.4. The proof given above implies that the claim of Theorem 2.2 remains true if we replace the condition of existence of interior points for the set $f\left(u, B_{u}\right)$ by the following one: for any $u \in U$, there is a point $x_{u} \in X$ and a finite-dimensional subspace $X_{u} \subset X$ such that the restriction of $D_{x} f\left(u, x_{u}\right)$ to $X_{u}$ is an isomorphism from $X_{u}$ onto $F$ (cf. Step 1 of the proof).

\section{Applications}

Throughout this section, we use the standard functional spaces $H$ and $V$ arising in the theory of Navier-Stokes equations; they are defined in Subsection 3.1. We shall also use the spaces

$$
\mathcal{X}=C\left(\mathbb{R}_{+}, H\right) \cap L_{\text {loc }}^{2}\left(\mathbb{R}_{+}, V\right), \quad \mathcal{X}_{T}=C\left(J_{T}, H\right) \cap L^{2}\left(J_{T}, V\right),
$$

where $T>0$ and $J_{T}=[0, T]$.

\subsection{Navier-Stokes equations perturbed by a time-discrete random force}

Let us consider the 2D Navier-Stokes (NS) system on the torus $\mathbb{T}^{2}=\mathbb{R}^{2} / 2 \pi \mathbb{Z}^{2}$. Define the spaces

$$
H=\left\{u \in L^{2}\left(\mathbb{T}^{2}, \mathbb{R}^{2}\right): \operatorname{div} u=0 \text { on } \mathbb{T}^{2}\right\}, \quad V=H^{1}\left(\mathbb{T}^{2}, \mathbb{R}^{2}\right) \cap H,
$$

endowed with natural norms. Here $H^{s}\left(\mathbb{T}^{2}, \mathbb{R}^{2}\right)$ denotes the space of vector functions $\left(u_{1}, u_{2}\right)$ whose components belong to the Sobolev space of order $s$. Let $\Pi: L^{2}\left(\mathbb{T}^{2}, \mathbb{R}^{2}\right) \rightarrow H$ be the orthogonal projection in $L^{2}\left(\mathbb{T}^{2}, \mathbb{R}^{2}\right)$ onto $H$. After applying the projection $\Pi$, the NS system reduces to the following evolution equation in $H$ :

$$
\dot{u}+v L u+B(u, u)=g(t, x),
$$

where $v>0$ is the viscosity, $L=-\Pi \Delta$, and $B(u, v)=\Pi((u, \nabla) v)$. In this subsection, we assume that

$$
g(t, x)=\sum_{k=1}^{\infty} I_{k, T}(t) \eta_{k}(x),
$$


where $T>0$ is a parameter, $I_{k, T}(t)$ is the indicator function of the time interval $[(k-1) T, k T)$, and $\left\{\eta_{k}\right\}$ is a sequence of $H$-valued i.i.d. random variables defined on a probability space $(\Omega, \mathcal{F}, \mathbb{P})$. Standard theorems on well-posedness of the 2D NS system (e.g., see [5]) imply that, for almost every $\omega \in \Omega$, problem (3.1), (3.2) has a unique solution $u \in \mathcal{X}$ that satisfies the initial condition

$$
u(0)=u_{0},
$$

where $u_{0} \in H$ is an arbitrary function. We shall denote by $S_{t}: H \rightarrow H$ the random operator that takes $u_{0}$ to $u(t)$. Our aim is to study the distribution for projections of the random variables $S_{k T}\left(u_{0}\right)$ to finite-dimensional subspaces of $H$.

Let $\left\{e_{j}\right\}$ be a complete set of eigenfunctions for $L$ indexed in an increasing order of the corresponding eigenvalues $\alpha_{j}$ and let $H_{N}$ be the vector space spanned by $e_{j}, j=1, \ldots, N$. We shall assume that the i.i.d. random variables $\eta_{k}$ satisfy the following condition.

(D) The random variables $\eta_{k}$ have the form

$$
\eta_{k}=\sum_{j=1}^{\infty} b_{j} \xi_{j k} e_{j},
$$

where $b_{j} \geqslant 0$ are some constants such that

$$
\sum_{j=1}^{\infty} b_{j}^{2}<\infty,
$$

and $\xi_{j k}$ are independent scalar random variables whose distribution $\pi_{j}$ possesses a continuous density with respect to the Lebesgue measure, and $\operatorname{supp} \pi_{j} \ni 0$ for any $j \geqslant 0$.

The following theorem shows that, under the above condition, all finite-dimensional projections of the distribution for $S_{k T}(u)$ have continuous densities with respect to the Lebesgue measure, provided that $k$ is sufficiently large and $T$ does not belong to a discrete exceptional set.

Theorem 3.1. Suppose that condition (D) is fulfilled. Then there is an integer $N \geqslant 1$ not depending on $v$ and $\left\{\eta_{k}\right\}$ such that the following two statements hold, provided that

$$
b_{j} \neq 0 \quad \text { for } j=1, \ldots, N \text {. }
$$

(i) For any constant $v>0$ and any finite-dimensional subspace $F \subset H$, there is a discrete subset $\boldsymbol{T}=\boldsymbol{T}(v, F) \subset$ $\mathbb{R}_{+} \backslash\{0\}$ such that if $T \notin \boldsymbol{T}$ and $R>0$, then for any $u \in B_{H}(R)$ and an appropriate integer $k=k(v, F, R) \geqslant 1$ the distribution of $\mathrm{P}_{F} S_{k T}(u)$ possesses a density with respect to $\ell_{F}$.

(ii) Let us set $\lambda_{u}(t)=\mathcal{D}\left(\mathrm{P}_{F} S_{t}(u)\right)$. Then $\lambda_{u}(k T)$ continuously depends on $u \in B_{H}(R)$ in the total variation norm.

Remark 3.2. It is possible to give a more precise description of the integer $N$ in (3.6). Namely, it is the minimal integer $N \geqslant 1$ such that the vectors $e_{1}, \ldots, e_{N}$ form a saturating set (see Section A.3 in Appendix A for a definition of a saturating set). In particular, if the eigenfunctions of $L$ are indexed in a suitable way, then one can take $N=6$.

Proof of Theorem 3.1. Step 1. We wish to apply Theorem 2.2 and Remark 2.4. Let $H_{0} \subset H$ be the subspace spanned by those vectors $e_{j}$ for which $b_{j} \neq 0$ and let $\boldsymbol{X}_{k}$ be the direct product of $k$ copies of $H_{0}$. We fix a finite-dimensional subspace $F \subset H$ and consider the operator

$$
f_{k}: \mathbb{R}_{+}^{*} \times H \times \boldsymbol{X}_{k} \rightarrow F, \quad\left(T, u_{0}, \eta_{1}, \ldots, \eta_{k}\right) \mapsto \mathrm{P}_{F} u(k T),
$$

where $\mathbb{R}_{+}^{*}=\mathbb{R}_{+} \backslash\{0\}$ and $u(t)$ denotes the solution of problem (3.1)-(3.3) in which $\left\{\eta_{k}\right\}$ is regarded as a sequence of deterministic functions in $H_{0}$. In view of Proposition A.2, the operator $f_{k}$ is analytic on $\mathbb{R}_{+}^{*} \times H \times \boldsymbol{X}_{k}$. Furthermore, if condition (D) is fulfilled, then for any integer $k \geqslant 1$ the distribution of the $\boldsymbol{X}_{k}$-valued random variable $\boldsymbol{\eta}_{k}=\left(\eta_{1}, \ldots, \eta_{k}\right)$ satisfies property $(\mathrm{P})$. Suppose we have established the existence of a discrete subset $\boldsymbol{T} \subset \mathbb{R}_{+}^{*}$ possessing the following property: 
(C) for any $R>0$ there is an integer $k \geqslant 1$ such that if $T \notin \boldsymbol{T}$ and $u_{0} \in B_{H}(R)$, then the derivative

$$
\left(D_{\boldsymbol{\eta}_{k}} f_{k}\right)\left(T, u_{0}, \boldsymbol{\eta}_{k}\right): \boldsymbol{X}_{k} \rightarrow F
$$

is surjective for at least one point $\boldsymbol{\eta}_{k}=\left(\eta_{1}, \ldots, \eta_{k}\right) \in \boldsymbol{X}_{k}$.

In this case, statements (i) and (ii) of the theorem are straightforward consequences of Theorem 2.1 and Remark 2.3 in which $X=X_{k}$ and $U=B_{H}(R)$.

Step 2. To prove (C), we first assume that $u_{0}=0$. Let us denote by $\mathcal{R}_{1}: L^{2}\left(J_{1}, H\right) \rightarrow H$ the operator that takes each function $g \in L^{2}\left(J_{1}, H\right)$ to $u(1, x)$, where $u \in \mathcal{X}_{1}$ is the solution of (3.1), (3.3) with $u_{0}=0$. By Proposition A.5, there is an integer $N \geqslant 1$ such that the Navier-Stokes system (3.1) with $g \in L^{2}\left(J_{1}, H_{N}\right)$ is solidly controllable in time 1 for the projection to $F$. (See Definition A.4 for the concept of solid controllability.) In particular, there is a compact subset $K \subset L^{2}\left(J_{1}, H\right)$ and a constant $\varepsilon>0$ such that $\Phi(K) \supset B_{F}(1)$ for any continuous map satisfying the inequality

$$
\sup _{g \in K}\left\|\Phi(g)-\mathrm{P}_{F} \mathcal{R}_{1}(g)\right\|_{F} \leqslant \varepsilon
$$

For any integer $m \geqslant 1$, denote by $Y_{m} \subset L^{2}\left(J_{1}, H_{N}\right)$ the subspace of functions that are constant on any interval of the form $\left[\frac{l-1}{m}, \frac{l}{m}\right), 1 \leqslant l \leqslant m$. It is clear that $\bigcup_{m} Y_{m}$ is dense in $L^{2}\left(J_{1}, H_{N}\right)$. Since $K$ is compact, for any $\delta>0$ we can find an integer $m \geqslant 1$ such that

$$
\sup _{g \in K}\left\|g-\mathrm{P}_{Y_{m}} g\right\|_{V} \leqslant \delta .
$$

It follows from the continuity of $\mathcal{R}_{1}$ that for any $\varepsilon>0$ there is an integer $m \geqslant 1$ such that (3.8) is satisfied for $\Phi(g)=\mathrm{P}_{F} \mathcal{R}_{1}\left(\mathrm{P}_{Y_{m}} g\right)$. This implies that

$$
\mathrm{P}_{F} \mathcal{R}_{1}\left(Y_{m}\right) \supset B_{F}(1) .
$$

Now note that if we denote by $I_{l, m}(t)$ the indicator function of the interval $\left[\frac{l-1}{m}, \frac{l}{m}\right)$ and identify the function

$$
g(t, x)=\sum_{l=1}^{m} I_{l, m}(t) \eta_{l}(x) \in Y_{m}
$$

with the vector $\boldsymbol{\eta}=\left(\eta_{1}, \ldots, \eta_{m}\right) \in \boldsymbol{X}_{m}$, then we can write

$$
f_{m}\left(m^{-1}, 0, \eta_{m}\right)=\mathrm{P}_{F} \mathcal{R}_{1}(g) \text {. }
$$

Combining (3.9) and (3.10), we conclude that there is a finite-dimensional subspace $X \subset X_{m}$ such that

$$
f_{m}\left(m^{-1}, 0, X\right) \supset B_{F}(1) .
$$

Sard's theorem now implies that the derivative

$$
\left(D_{\boldsymbol{\eta}_{m}} f_{m}\right)\left(m^{-1}, 0, \boldsymbol{\eta}_{m}\right): \boldsymbol{X}_{m} \rightarrow F
$$

is surjective for at least one point $\boldsymbol{\eta}_{m}^{0} \in \boldsymbol{X}_{m}$. Since $\left(D_{\boldsymbol{\eta}_{m}} f_{m}\right)\left(T, 0, \boldsymbol{\eta}_{m}^{0}\right)$ is an analytic function with respect to $T>0$, we see that there is a discrete set $\boldsymbol{T} \subset \mathbb{R}_{+}^{*}$ such that

$$
\left(D_{\boldsymbol{\eta}_{m}} f_{m}\right)\left(T, 0, \boldsymbol{\eta}_{m}^{0}\right): \boldsymbol{X}_{m} \rightarrow F \quad \text { is surjective for any } T \notin \boldsymbol{T} .
$$

Step 3. We can now verify property (C). Let us fix any $T \notin \boldsymbol{T}$ and $R>0$. We claim that if an integer $l \geqslant 1$ is sufficiently large and $k=l+m$, then the linear operator (3.7) is surjective for $\boldsymbol{\eta}_{k}=\left(0, \ldots, 0, \boldsymbol{\eta}_{m}^{0}\right)$ and any $u_{0} \in B_{H}(R)$. Indeed, the definition of $f_{k}$ implies that

$$
f_{k}\left(T, u_{0}, \boldsymbol{\eta}_{k}^{0}\right)=f_{m}\left(T, u(l T), \boldsymbol{\eta}_{m}^{0}\right)
$$

where $u(t)$ is the solution of (3.1), (3.3) with $g \equiv 0$. It follows that

$$
\operatorname{Im}\left\{\left(D_{\boldsymbol{\eta}_{k}} f_{k}\right)\left(T, u_{0}, \boldsymbol{\eta}_{k}^{0}\right)\right\} \supset \operatorname{Im}\left\{\left(D_{\boldsymbol{\eta}_{m}} f_{m}\right)\left(T, u(l T), \boldsymbol{\eta}_{m}^{0}\right)\right\},
$$


where $\operatorname{Im}\{A\}$ denotes the image of a linear operator $A$. In view of (3.11) and the continuity of $D_{\eta_{m}} f_{m}$, we can find $r>0$ such that

$$
\operatorname{Im}\left\{\left(D_{\boldsymbol{\eta}_{m}} f_{m}\right)\left(T, v, \boldsymbol{\eta}_{m}^{0}\right)\right\}=F \quad \text { for any } v \in B_{H}(r) .
$$

Furthermore, the dissipation property of the homogeneous Navier-Stokes system implies that

$$
u(l T) \in B_{H}(r) \quad \text { for any } u_{0} \in B_{H}(R),
$$

where $l=l(R, T) \geqslant 1$ is sufficiently large. Combining (3.12)-(3.14), we arrive at the required result.

Remark 3.3. Analysing the proof given above, it is possible to establish the following property, which shows that the "bad" subset $\boldsymbol{T}$ constructed in Theorem 3.1 cannot accumulate to zero if we allow the integer $k$ to depend on $T$ :

- For any constant $v>0$ and any finite-dimensional subspace $F \subset H$ there is $T_{0}>0$ such that if $T \in\left(0, T_{0}\right]$ and $R>0$, then for any $u \in B_{H}(R)$ and an appropriate integer $k=k(v, F, R, T) \geqslant 1$ the distribution of $\mathrm{P}_{F} S_{k T}(u)$ possesses a density with respect to $\ell_{F}$ and continuously depends on $u$ in the total variation norm.

We now study stationary solutions of (3.1), (3.2). Since $\left\{\eta_{k}\right\}$ are i.i.d. random variables in $H$, for any deterministic initial function $u_{0}$ the sequence $\{u(k T), k \geqslant 0\}$ is a Markov chain. Thus, the set of all solutions restricted to the times $k T$ form a Markov family in $H$. Let $P_{k}(u, \Gamma)$ be the corresponding transition function and let $\mathfrak{P}_{k}^{*}$ be the Markov operator associated with $P_{k}$. Using standard a priori estimates for solutions of the Navier-Stokes system and applying the Bogolyubov-Krylov argument (e.g., see [12]), one can show that $\mathfrak{P}_{k}^{*}$ has at least one stationary distribution $\mu$ :

$$
\mathfrak{P}_{k}^{*} \mu=\mu \text { for all } k \geqslant 1 \text {. }
$$

A simple consequence of Theorem 3.1 is the following result:

Corollary 3.4. Suppose that the conditions of Theorem 3.1 are fulfilled. Let $F \subset H$ be a finite-dimensional subspace and let $T \notin \boldsymbol{T}(\nu, F)$. Then for any stationary measure $\mu$ the projection $\mathrm{P}_{F} \mu$ possesses a density with respect to the Lebesgue measure on $F$.

Proof. Let $\Gamma \subset F$ be a Borel set of zero Lebesgue measure and let $\varepsilon>0$. Choose a constant $R>0$ so large that

$$
\mu\left(B_{H}(R)\right) \geqslant 1-\varepsilon .
$$

By assertion (i) of Theorem 3.1, we can find an integer $k \geqslant 1$ such that

$$
\left(\mathrm{P}_{F} P_{k}\right)(u, \Gamma)=0 \quad \text { for all } u \in B_{H}(R) .
$$

Combining (3.15)-(3.17), we derive

$$
\mathrm{P}_{F} \mu(\Gamma)=\int_{H}\left(\mathrm{P}_{F} P_{k}\right)(u, \Gamma) \mu(\mathrm{d} u)=\int_{B_{H}^{c}(R)}\left(\mathrm{P}_{F} P_{k}\right)(u, \Gamma) \mu(\mathrm{d} u) \leqslant \mu\left(B_{H}^{c}(R)\right) \leqslant \varepsilon,
$$

where $B_{H}^{c}(R)$ denotes the complement of $B_{H}(R)$. Since $\varepsilon>0$ was arbitrary, we conclude that $\mathrm{P}_{F} \mu(\Gamma)=0$. This completes the proof of the corollary.

\subsection{Navier-Stokes equations with a non-degenerate random perturbation}

In this subsection, we consider the NS system (3.1) with a right-hand side of the form

$$
g(t, x)=h(t, x)+\eta(t, x) .
$$

We assume that $h \in L_{\text {loc }}^{2}\left(\mathbb{R}_{+}, H\right)$ is a deterministic function and $\eta$ is a random process whose trajectories belong to $L_{\text {loc }}^{2}\left(\mathbb{R}_{+}, H_{0}\right)$, where $H_{0} \subset H$ is a closed subspace. Denote by $\mu_{T}, T>0$, the distribution of the restriction of $\eta$ to the interval $J_{T}$ and by $S_{t}: H \rightarrow H$ the random operator that takes each $u_{0} \in H$ to $u(t)$, where $u \in \mathcal{X}$ is the solution of (3.1), (3.3), (3.18). 
Theorem 3.5. There is an integer $N \geqslant 1$ not depending on $v>0$ such that if $h \in L_{\mathrm{loc}}^{2}\left(\mathbb{R}_{+}, H\right)$ is a given function, $T>0$ is a constant, $H_{0} \subset H$ is a subspace containing $H_{N}$, and $\mu_{T}$ is a decomposable measure on $L^{2}\left(J_{T}, H_{0}\right)$ satisfying property $(\mathrm{P})$, then for any $u_{0} \in H$ and any positive $t \in J_{T}$ the following statements take place. ${ }^{2}$

(i) Let $F \subset H$ be a finite-dimensional subspace and let $\lambda_{u}(t)$ be the distribution of $\mathrm{P}_{F} S_{t}(u)$. Then $\lambda_{u}(t) \ll \ell_{F}$.

(ii) The measure $\lambda_{u}(t)$ continuously depends on $u \in H$ in the total variation norm.

Proof. Both assertions are straightforward consequences of Theorem 2.2 and Propositions A.2 and A.5. Indeed, let $f_{t}: H \times L^{2}\left(J_{T}, H\right) \rightarrow F$ be the operator that takes each pair $\left(u_{0}, \eta\right)$ to $\mathrm{P}_{F} u(t)$, where $u \in \mathcal{X}_{T}$ is the solution of problem (3.1), (3.3), (3.18) with deterministic functions $h$ and $\eta$. By assumption, the distribution $\mu_{T}$ of the restriction of $\eta$ to $J_{T}$ satisfies condition (P), and by Proposition A.2, the operator $f_{t}$ is analytic with respect to $\left(u_{0}, \eta\right)$. Furthermore, taking into account Proposition A.5 and repeating the argument used in Section 3.1, for any $u \in H$ and any positive $t \in J_{T}$ we can find a ball $B_{u}$ in a finite-dimensional subspace $X_{u} \subset L^{2}\left(J_{T}, H\right)$ such that $f_{t}\left(u, B_{u}\right) \subset F$ has at least one interior point. Thus, the conditions of Theorem 2.2 are fulfilled, and we can conclude that assertions (i) and (ii) hold.

Example 3.6. Let us consider an example of a random force $\eta(t, x)$ for which the hypotheses of Theorem 3.5 are satisfied for any $T>0$. Let $H_{0} \subset H$ be any finite-dimensional subspace and let $\{\eta(t), t \geqslant 0\}$ be a homogeneous Gaussian process in $H_{0}$ with a correlation function $K(t)$. (For the existence of such a process, see [11, Chapter 3].) Suppose that $K(t)$ is a positive-definite operator for any $t \geqslant 0$. Then, for any $T>0$, the distribution of the restriction of $\eta$ to $J_{T}$ is a non-degenerate Gaussian measure. As is explained in Example 1.4, such a measure satisfies property (P).

\subsection{Navier-Stokes equations perturbed by a white noise}

This subsection is devoted to studying the NS system (3.1), (3.18), in which $h \in L_{\text {loc }}^{2}\left(\mathbb{R}_{+}, H\right)$ is a deterministic function and $\eta(t, x)$ is a random process white in time and $H^{2}$-regular in the space variables. More precisely, we define the space $U=V \cap H^{2}\left(\mathbb{T}^{2}, \mathbb{R}^{2}\right)$ endowed with the $H^{2}$-norm and assume that there is a non-negative nuclear operator $Q \in \mathcal{L}(U)$ such that

$$
\eta(t, x)=\frac{\partial}{\partial t} \zeta(t, x)
$$

where $\zeta$ is a Gaussian process in $U$ with continuous trajectories and the covariance operator

$$
K(t, s)=(t \wedge s) Q, \quad t, s \geqslant 0 .
$$

It follows that

$$
\zeta(t, x)=\sum_{j=1}^{\infty} b_{j} \beta_{j}(t) g_{j}(x),
$$

where $\left\{g_{j}\right\}$ is an orthonormal basis in $U$ formed of the eigenvectors of $Q, b_{j}^{2}$ is the eigenvalue of $Q$ corresponding to $g_{j}$, and $\left\{\beta_{j}\right\}$ is a sequence of independent standard Brownian motions. It is well known (see [21,10]) that for almost every value of the random parameter the Cauchy problem for (3.1), (3.18), (3.19) has a unique solution in the space $\mathcal{X}$, and we denote by $S_{t}: H \rightarrow H$ its resolving (random) operator.

Theorem 3.7. There is an integer ${ }^{3} N \geqslant 1$ not depending on $v>0$ such that if $h \in L_{\text {loc }}^{2}\left(\mathbb{R}_{+}, H\right)$ is a given function and the image of $Q$ contains $H_{N}$, then for any $u_{0} \in H$ and $t>0$ assertions (i) and (ii) of Theorem 3.5 hold, and

$$
\operatorname{supp} \lambda_{u}(t)=F \text {. }
$$

\footnotetext{
2 See Remark 3.2 for a more precise description of $N$.

3 A more precise description of $N$ can be found in Remark 3.2.
} 
Proof. Step 1. Let us fix $T>0$ and study our problem on the interval $J_{T}$. The solution of (3.1), (3.3), (3.18), (3.19) can be represented in the form $u=v+\zeta$, where $v \in \mathcal{X}_{T}$ is a solution of the problem

$$
\dot{v}+v L v+B(v, v)+B(v, \zeta)+B(\zeta, v)=h(t)-v L \zeta-B(\zeta, \zeta), \quad v(0)=u_{0} .
$$

Let $\mathcal{F}: H \times L^{2}\left(J_{T}, U\right) \rightarrow \mathcal{X}_{T}$ be the operator that takes $\left(u_{0}, \zeta\right)$ to the solution $v \in \mathcal{X}_{T}$ of (3.21). Using standard methods of the theory of 2D NS equations, one can show that $\mathcal{F}$ is well defined and analytic (cf. Proposition A.2). Thus, denoting by $\mathcal{F}_{t}\left(u_{0}, \zeta\right)$ the restriction of $\mathcal{F}\left(u_{0}, \zeta\right)$ to the time $t$, we can write

$$
\mathrm{P}_{F} u(t)=\mathrm{P}_{F} \zeta(t)+\mathrm{P}_{F} \mathcal{F}_{t}\left(u_{0}, \zeta\right)=: f_{t}\left(u_{0}, \zeta\right) .
$$

It follows that $\lambda_{u_{0}}(t)=\mathcal{D}\left(\mathrm{P}_{F} u(t)\right)$ is the image of $\mu_{T}$ under the analytic map $f_{t}\left(u_{0}, \cdot\right)$, where $\mu_{T}$ is the distribution of the restriction of $\zeta$ to $J_{T}$. Hence, assertions (i) and (ii) of Theorem 3.5 will be established if we show that the hypothesis of Theorem 2.2 are satisfied.

Step 2. Let us regard $\mu_{T}$ as a measure on $L^{2}\left(J_{T}, U_{0}\right)$, where $U_{0}$ denote the closure in $U$ of the image of $Q$. In this case, $\mu_{T}$ is a non-degenerate Gaussian measure that satisfies property (P) (see Examples 1.2 and 1.4). We claim that for any $u_{0} \in H, t \in J_{T} \backslash\{0\}$, and $r>0$ there is a ball $B_{u_{0}}=B_{u_{0}}(t, r)$ in a finite-dimensional subspace of $L^{2}\left(J_{T}, H_{N}\right)$ such that

$$
f_{t}\left(u_{0}, B_{u_{0}}\right) \supset B_{F}(r) .
$$

Indeed, for any deterministic function $\xi \in C^{1}\left(J_{T}, H_{N}\right)$, we can write

$$
u(t)=\xi(t)+\mathcal{F}_{t}\left(u_{0}, \xi\right)=\mathcal{R}_{t}\left(u_{0}, \hat{\xi}\right),
$$

where $\mathcal{R}_{t}$ is the resolving operator for the NS system (see Section A.3) and $\hat{\xi}=\partial_{t} \xi$. It follows from Proposition A.5 (cf. Step 2 in the proof of Theorem 3.1) that if $N \geqslant 1$ is sufficiently large, then there is a ball $\widehat{B}_{u_{0}}$ in a finite-dimensional subspace of $C\left(J_{T}, H_{N}\right)$ such that

$$
\mathrm{P}_{F} \mathcal{R}_{t}\left(u_{0}, \widehat{B}_{u_{0}}\right) \supset B_{F}(r) \text {. }
$$

It is clear that the image of $\widehat{B}_{u_{0}}$ under the linear operator $\hat{\xi} \mapsto \int_{0}^{\cdot} \hat{\xi}(s) \mathrm{d} s$ is contained in a finite-dimensional ball $B_{u_{0}}$. Combining this with (3.23) and (3.24), we arrive at (3.22). Hence, Theorem 2.2 is applicable, and we obtain assertions (i) and (ii).

Step 3. Relation (3.20) is a standard consequence of controllability, and we confine ourselves to outlining its proof. Let $y \in F$ be an arbitrary point and $\varepsilon>0$. We wish to show that

$$
\lambda_{u_{0}}(t)\left(B_{F}(y, \varepsilon)\right)>0 .
$$

In view of (3.22), there is $\xi_{0} \in L^{2}\left(J_{T}, H_{N}\right)$ such that $f_{t}\left(u_{0}, \xi_{0}\right)=y$. By continuity, there is $\delta>0$ such that if

$$
\left\|\xi-\xi_{0}\right\|_{L^{2}\left(J_{T}, U\right)} \leqslant \delta
$$

then $\left\|f_{t}\left(u_{0}, \xi\right)-f_{t}\left(u_{0}, \xi_{0}\right)\right\|_{F} \leqslant \varepsilon$. Since $\mu_{T}$ is non-degenerate, it follows from (1.7) that

$$
\mu_{T}\left(\left\{\xi \in L^{2}\left(J_{T}, U_{0}\right): \xi \text { satisfies }(3.26)\right\}\right)>0 \text { for any } \delta>0 .
$$

This implies the required property (3.25). The proof is complete.

Remark 3.8. It is established in [2] that the 2D Euler equations considered in the space $H^{s}\left(\mathbb{T}^{2}, \mathbb{R}^{2}\right)$ with $s \geqslant 2$ possess the property of exact controllability for observed projections (cf. Section A.3). Using this fact, one can show that some results similar to those in Theorems 3.5 and 3.7 are true for the Euler equations. This will be done in a forthcoming publication.

As in the case of the NS system perturbed by the piecewise-constant random force (3.2), Theorem 3.7 readily implies the existence of a density with respect to the Lebesgue measure for finite-dimensional projections of any stationary distribution. Namely, consider Eq. (3.1) with the right-hand side

$$
g(t, x)=h(x)+\eta(t, x),
$$

where $h \in H$ is a deterministic function and $\eta$ is a random process of the form (3.19). In this case, problem (3.1), (3.27) generates a Markov family in the space $H$, which has at least one stationary measure (e.g., see [10]). The proof of the following result is similar to that of Corollary 3.4 and will be omitted. 
Corollary 3.9. Under the conditions of Theorem 3.7, for any deterministic function $h \in H$, any stationary measure $\mu$, and any finite-dimensional subspace $F \subset H$, the projection $\mathrm{P}_{F} \mu$ possesses an almost everywhere positive density with respect to the Lebesgue measure on $F$.

\section{Appendix A}

\section{A.1. Small perturbations of smooth functions}

Let $E$ and $F$ be finite-dimensional vector spaces, let $D \subset E$ be an open subset, and let $f: D \rightarrow F$ be an infinitely differentiable function.

Proposition A.1. Suppose that $f(D)$ has a non-empty interior. Then there is $\varepsilon>0$ such that if $g: D \rightarrow F$ is a continuous function satisfying the inequality

$$
\sup _{x \in D}\|f(x)-g(x)\|_{F} \leqslant \varepsilon,
$$

then $g(D)$ has a non-empty interior.

Proof. Since the interior of $f(D)$ is non-empty, Sard's theorem (see [19]) implies that $f$ has at least one regular point $x_{0} \in D$. Let us set $y_{0}=f\left(x_{0}\right)$ and assume, without loss of generality, that $x_{0}=0$ and $y_{0}=0$. We can represent $E$ as a direct sum $E=E_{1}+E_{2}$ such that the restriction of the derivative $D f\left(x_{0}\right)$ to $E_{1}$ is an isomorphism from $E_{1}$ onto $F$. The required assertion will be proved if we show that for any continuous function $g: D \rightarrow F$ satisfying (A.1) with $\varepsilon \ll 1$ the interior of $g\left(D \cap E_{1}\right)$ is non-empty. To this end, denote by $f_{1}$ and $g_{1}$ the restriction of $f$ and $g$ to $D \cap E_{1}$ and note that if $\delta>0$ is sufficiently small, then $\operatorname{deg}_{\delta} f_{1}=1$, where $\operatorname{deg}_{\delta} h$ denotes the degree of a continuous function $h: D \cap E_{1} \rightarrow F$ with the respect to the ball $B_{E_{1}}(\delta)$. It follows that if $g$ satisfies (A.1) with sufficiently small $\varepsilon$, then $\operatorname{deg}_{\delta} g_{1}=\operatorname{deg}_{\delta} f_{1}$. Since $y=0$ is a regular point of $f_{1}$, we can find $r>0$ such that any point $y \in F$ with norm not exceeding $r$ has a preimage under $g_{1}$. This shows that the interior of $g_{1}(D)$ is non-empty. The proposition is proved.

\section{A.2. Resolving operator for the Navier-Stokes equations}

Let us consider the Navier-Stokes system (3.1) supplemented with the initial condition (3.3). It is well known [5] that if $u_{0} \in H$ and $g \in L^{1}\left(J_{T}, H\right)$ for some $T>0$, then problem (3.1), (3.3) has a unique solution

$$
u_{v} \in \mathcal{X}_{T}:=C\left(J_{T}, H\right) \cap L^{2}\left(J_{T}, V\right) .
$$

Let $\mathbb{R}_{+}^{*}=(0, \infty)$ and let $\mathcal{R}: \mathbb{R}_{+}^{*} \times H \times L^{1}\left(J_{T}, H\right) \rightarrow \mathcal{X}_{T}$ be an operator that takes $\left(\nu, u_{0}, f\right)$ to the solution $u_{v} \in \mathcal{X}_{T}$. The following result on analyticity of $\mathcal{R}$ is established ${ }^{4}$ in [14].

Proposition A.2. The operator $\mathcal{R}$ is analytic on the domain of its definition.

We now consider the problem (3.1)-(3.3), in which $T>0$ is a parameter and $\left\{\eta_{k}\right\} \subset H$ is a sequence of deterministic functions. For any integer $k \geqslant 1$, we set $\boldsymbol{H}_{k}=H \times \cdots \times H$ ( $k$ times) and define the operator

$$
\mathcal{S}_{k}: \mathbb{R}_{+}^{*} \times H \times \boldsymbol{H}_{k} \rightarrow H, \quad\left(T, u_{0}, \boldsymbol{\eta}_{k}\right) \rightarrow u(k T),
$$

where $\boldsymbol{\eta}_{k}=\left(\eta_{1}, \ldots, \eta_{k}\right) \in \boldsymbol{H}_{k}$ and $u(t)$ denotes the solution of (3.1)-(3.3).

Corollary A.3. For any integer $k \geqslant 1$, operator (A.2) is analytic on the domain of its definition.

\footnotetext{
4 In [14], the analyticity of $\mathcal{R}$ is proved for a fixed $v>0$. However, an unessential modification of the proof enables one to handle the general
} case. 
Proof. Let us rewrite (3.1) in the form

$$
\dot{u}+v L u+B(u, u)=\sum_{k=1}^{\infty} I_{[k-1, k)}(t / T) \eta_{k}(x),
$$

where $I_{[k-1, k)}$ stands for the indicator function of $[k-1, k)$. Performing the change of variables $u(t)=T^{-1} v(s)$, $s=t / T$, in Eqs. (A.3) and (3.3), we arrive at the problem

$$
\partial_{s} v+T v L v+B(v, v)=T^{2} \hat{g}(s, x), \quad v(0)=T u_{0},
$$

where $\hat{g}(s, x)=\sum_{k} I_{[k-1, k)}(s) \eta_{k}(x)$. It follows that

$$
\mathcal{S}_{k}\left(T, u_{0}, \eta_{k}\right)=T^{-1} \mathcal{R}_{k}\left(T \nu, T u_{0}, T^{2} \hat{g}\right),
$$

where $\mathcal{R}_{t}\left(v, u_{0}, f\right)$ denotes the restriction of $\mathcal{R}\left(v, u_{0}, f\right)$ to the time $t$. The required result follows now from Proposition A.2.

\section{A.3. A controllability property of the Navier-Stokes equations}

Recall that $J_{T}=[0, T]$ and that $H_{N} \subset H$ denotes the vector space spanned by the first $N$ eigenfunctions of the Stokes operator $L$. Consider the Navier-Stokes system (3.1), (3.18) where $h \in L^{2}\left(J_{T}, H\right)$ is a given function and $\eta \in L^{2}\left(J_{T}, H_{N}\right)$ is a control function. In this subsection, we assume that the viscosity $v>0$ and the function $h$ are fixed and denote by $\mathcal{R}_{t}: H \times L^{2}\left(J_{T}, H\right) \rightarrow H$ the operator that takes $\left(u_{0}, \eta\right)$ to the function $u(t)$, where $u \in \mathcal{X}_{T}$ is the solution of (3.1), (3.3), (3.18).

Let $F \subset H$ be a finite-dimensional subspace, let $\mathrm{P}_{F}: H \rightarrow F$ be the orthogonal projection in $H$ onto $F$, and let $T>0$ be a constant.

Definition A.4. Problem (3.1), (3.18) is said to be controllable in time $T$ for the projection to $F$ if

$$
\mathrm{P}_{F} \mathcal{R}_{T}\left(u_{0}, L^{2}\left(J_{T}, H_{N}\right)\right) \supset F
$$

for any $u_{0} \in H$.

Problem (3.1), (3.18) is said to be solidly controllable in time $T$ for the projection to $F$ if for any $R>0$ and $u_{0} \in H$ there is a constant $\varepsilon>0$ and a compact subset $K=K\left(R, u_{0}\right) \subset L^{2}\left(J_{T}, H_{N}\right)$ such that $\Phi(K) \supset B_{F}(R)$, where $\Phi: K \rightarrow F$ is an arbitrary continuous map satisfying the inequality

$$
\sup _{\eta \in K}\left\|\Phi(\eta)-\mathrm{P}_{F} \mathcal{R}_{T}\left(u_{0}, \eta\right)\right\|_{F} \leqslant \varepsilon
$$

Proposition A.5. There is an integer $N \geqslant 1$ not depending on $v$ and $h$ such that for any $T>0$ and any finitedimensional subspace $F \subset H$ the Navier-Stokes system (3.1), (3.18) is solidly controllable in time $T$ for the projection to $F$.

In the case $h \equiv 0$, this result is established in [1,2]. The general situation can be treated by the same argument.

We now give a more precise description of the integer $N$ entering Proposition A.5. To this end, it is convenient to index the trigonometric basis in $H$ by the elements of $\mathbb{Z}^{2}$. Namely, we write $j=\left(j_{1}, j_{2}\right) \in \mathbb{Z}^{2}$ and set

$$
\begin{array}{ll}
e_{j}(x)=\sin (j x) j^{\perp} & \text { for } j_{1}>0 \text { or } j_{1}=0, j_{2}>0, \\
e_{j}(x)=\cos (j x) j^{\perp} & \text { for } j_{1}<0 \text { or } j_{1}=0, j_{2}<0, \\
e_{0}^{1}(x)=(1,0), & e_{0}^{2}(x)=(0,1),
\end{array}
$$

where $j^{\perp}=\left(-j_{2}, j_{1}\right)$. The family $\mathcal{E}=\left\{e_{0}^{i}, e_{j}, i=1,2, j \in \mathbb{Z}^{2} \backslash\{0\}\right\}$ is a complete set of eigenfunctions for the Stokes operator $L$ and, hence, is an orthogonal basis in $H$.

For any symmetric subset $\mathcal{K} \subset \mathbb{Z}^{2}$ containing the point $(0,0)$, we write $\mathcal{K}_{0}=\mathcal{K}$ and define $\mathcal{K}^{i}$ with $i \geqslant 1$ as the union of $\mathcal{K}^{i-1}$ and the family of vectors $l \in \mathbb{Z}^{2}$ for which there are $m, n \in \mathcal{K}^{i-1}$ such that

$$
l=m+n, \quad|m| \neq|n|, \quad m \wedge n \neq 0,
$$

where $m \wedge n=m_{1} n_{2}-m_{2} n_{1}$. 
Definition A.6. A symmetric subset $\mathcal{K} \subset \mathbb{Z}^{2}$ containing $(0,0)$ is said to be saturating if $\bigcup_{i \geqslant 0} \mathcal{K}^{i}=\mathbb{Z}^{2}$.

For any subset $\mathcal{K} \subset \mathbb{Z}^{2}$, we denote by $H_{\mathcal{K}}$ the vector space spanned by the family $\left\{e_{0}^{1}, e_{0}^{2}, e_{j}, j \in \mathcal{K} \backslash 0\right\}$. The following result is a refined version of Proposition A.5 (see [1,2]).

Proposition A.7. Let $\mathcal{K} \subset \mathbb{Z}^{2}$ be a saturating subset. Then for any positive constants $v$ and $T$, any function $h \in$ $L^{2}\left(J_{T}, H\right)$, and any finite-dimensional subspace $F \subset H$, the Navier-Stokes system (3.1), (3.18) with $\eta \in L^{2}\left(J_{T}, H_{\mathcal{K}}\right)$ is solidly controllable in time $T$ for the projection to $F$.

\section{References}

[1] A.A. Agrachev, A.V. Sarychev, Navier-Stokes equations: controllability by means of low modes forcing, J. Math. Fluid Mech. 7 (1) (2005) $108-152$.

[2] A.A. Agrachev, A.V. Sarychev, Controllability of 2D Euler and Navier-Stokes equations by degenerate forcing, Comm. Math. Phys. 265 (2006) 673-697.

[3] V.I. Bogachev, Gaussian Measures, Mathematical Surveys and Monographs, vol. 62, American Mathematical Society, Providence, RI, 1998.

[4] V. Bally, E. Pardoux, Malliavin calculus for white noise driven parabolic SPDEs, Potential Anal. 9 (1) (1998) $27-64$.

[5] P. Constantin, C. Foias, Navier-Stokes Equations, University of Chicago Press, Chicago, IL, 1988.

[6] R.C. Dalang, N.E. Frangos, The stochastic wave equation in two spatial dimensions, Ann. Probab. 26 (1) (1998) 187-212.

[7] S. Dineen, Zero one laws for probability measures on locally convex spaces, Math. Ann. 243 (2) (1979) 95-102.

[8] J.-P. Eckmann, M. Hairer, Uniqueness of the invariant measure for a stochastic PDE driven by degenerate noise, Comm. Math. Phys. 219 (2001) 523-565.

[9] W. Feller, An Introduction to Probability Theory and Its Applications, vol. II, John Wiley \& Sons, New York, 1971.

[10] F. Flandoli, Dissipativity and invariant measures for stochastic Navier-Stokes equations, NoDEA 1 (1994) $403-426$.

[11] I.I. Gihman, A.V. Skorohod, The Theory of Stochastic Processes. I, Springer-Verlag, Berlin, 1980.

[12] R.Z. Has'minskiŭ, Stochastic Stability of Differential Equations, Sijthoff \& Noordhoff, Alphen aan den Rijn, 1980.

[13] D. Henry, Geometric Theory of Semilinear Parabolic Equations, Lecture Notes in Mathematics, vol. 840, Springer-Verlag, Berlin, 1981.

[14] S.B. Kuksin, Diffeomorphisms of function spaces that correspond to quasilinear parabolic equations, Mat. Sb. (N.S.) 117 (159) (1982) 359$378,431$.

[15] J.A. León, D. Nualart, R. Pettersson, The stochastic Burgers equation: finite moments and smoothness of the density, Infin. Dimens. Anal. Quantum Probab. Relat. Top. 3 (3) (2000) 363-385.

[16] J. Mattingly, E. Pardoux, Malliavin calculus for the stochastic 2D Navier-Stokes equation, Comm. Pure Appl. Math. 59 (12) (2006) 17421790.

[17] D. Nualart, The Malliavin Calculus and Related Topics, Springer-Verlag, New York, 1995.

[18] D. Ocone, Stochastic calculus of variations for stochastic partial differential equations, J. Funct. Anal. 79 (2) (1988) $288-331$.

[19] S. Sternberg, Lectures on Differential Geometry, Chelsea Publishing Co., New York, 1983.

[20] R. Temam, Navier-Stokes Equations, North-Holland, Amsterdam, 1979.

[21] M.I. Vishik, A.V. Fursikov, Mathematical Problems in Statistical Hydromechanics, Kluwer, Dordrecht, 1988. 